\title{
Archéopages
}

Archéopages

Archéologie et société

Hors-série 1 | 2008

Construction $^{s}$ de l'archéologie

\section{Sur la genèse de la convention de Malte}

\author{
Willem J. H. Willems
}

\section{(2) OpenEdition}

Journals

Édition électronique

URL : https://journals.openedition.org/archeopages/888

DOI : 10.4000/archeopages.888

ISSN : 2269-9872

Éditeur

INRAP - Institut national de recherches archéologiques préventives

Édition imprimée

Date de publication : 1 février 2008

Pagination : 126-129

ISSN : 1622-8545

Référence électronique

Willem J. H. Willems, "Sur la genèse de la convention de Malte », Archéopages [En ligne], Hors-série 1 | 2008, mis en ligne le 01 février 2008, consulté le 25 février 2023. URL : http://

journals.openedition.org/archeopages/888 ; DOI : https://doi.org/10.4000/archeopages.888

Tous droits réservés 
L'exemple francilien n'est sans doute pas unique. Il fait déjà écho à une situation analogue en Picardie (Dubouloz et al. 2005). En bref, si la révolution néolithique a eu lieu en 5000 avant notre ère, celle des études sur le Néolithique se doit d'être permanente...

Constantin C., Mordant D., SimoninD. 1997: La Culture de Cerny, nouvelle économie, nouvelle société au Néolithique, actes du colloque international de Nemours, 9-11 mai 1994, Nemours, ApRAIF.

Chambon P., Mordant D., Pariat J.-G. 2003: «Sépultures du Néolithique moyen en Bassin parisien: le cas des architectures sépulcrales», in Actes du colloque interrégional sur le Néolithique, Luxembourg, p.9-31.

Demoule J.-P. (dir.) 2007: La Révolution néolithique en France, La Découverte, Paris.

Dubouloz J., Bostyn F., Chartier M., Cottiaux R., Le Bolloch M. 2005: «La recherche archéologique sur le Néolithique en Picardie», in «La recherche archéologique en Picardie: bilans et perspectives, journées d'études tenues à Amiens les 21 et 22 mars 2005", Revue archéologique de Picardie, $\mathrm{n}^{\circ} 3-4$, p.63-98.

\section{Sur la genèse de la convention de Malte}

Willem J. H. Willems

Université de Leyde, faculté d'Archéologie

$\mathbf{L}$ es différents systèmes de mise en application de la Convention européenne sur la protection du patrimoine archéologique ${ }^{\mathbf{1}}$ font, depuis plusieurs années, l'objet de débats au sein de la communauté archéologique. Indépendamment de la manière dont elle est appliquée par les différents pays, il est clair que la convention de Malte a occasionné partout des changements considérables dans la pratique de l'archéologie.

Des différences subsistent entre les systèmes entièrement ou partiellement privatisés et ceux dans lesquels l'État exerce encore un monopole sur l'archéologie. Mais les changements fondamentaux ont été les mêmes partout en Europe. Les archéologues universitaires sont à présent minoritaires; quasiment toute l'archéologie - y compris dans les systèmes non commerciaux - se pratique aujourd'hui sous contrat, d'où une inquiétude générale relative à sa pertinence, voire à sa qualité intrinsèque (Willems, Van den Dries 2007). Dans le même temps, les ressources de l'archéologie en général ont atteint des niveaux sans précédent, la conservation in situ des sites archéologiques étant devenue dans bien des cas une option réaliste qui répond à un intérêt croissant du public.

Le mérite en revient directement ou indirectement au succès politique de la convention de Malte et aux effets de son application.

La création de cette convention ne doit évidemment rien au hasard. Dans ce texte, il me semble intéressant d'évoquer brièvement les circonstances qui ont précédé sa signature en 1992, les raisons qui l'ont motivée et la façon dont les choses se sont déroulées. ${ }^{2}$

L'historique de la convention. En 1988, le Conseil de l'Europe fondait un nouveau comité intitulé «Comité restreint d'experts du Conseil de l'Europe sur l'archéologie et l'aménagement». Il avait pour mission de préparer une révision de la convention de Londres de 1969, qui avait été la première convention européenne pour la protection du patrimoine archéologique, mais qui était perçue comme inefficace et inadaptée face aux nouveaux défis rencontrés par l'archéologie dans les années 1980.

L'origine de ce développement remonte aux années 1960, lorsque les préoccupations environnementales acquirent de l'importance à la suite de la publication du rapport du Club de Rome. Très vite, il était apparu que le danger qui menaçait les ressources naturelles pesait également sur les ressources culturelles, lesquelles nécessitaient une gestion particulièrement attentive. Cette prise de conscience donna naissance à la gestion des ressources archéologiques dans le sens moderne du terme. Dans l'ensemble du monde occidental, les notions alors dominantes de préservation historique par le biais de la protection des monuments anciens et des «antiquités nationales» ont graduellement cédé le pas à des conceptions plus dynamiques d'une gestion des ressources archéologiques intégrée dès le départ aux systèmes de planification de l'espace urbain et rural sujet à des transformations de plus en plus rapides. Parti des États-Unis dans les années 1970, le mouvement a gagné une décennie plus tard de nombreux pays européens; depuis, il s'est répandu un peu partout dans le monde.

En Europe, la menace que l'effort de reconstruction de l'après-guerre faisait peser sur les ressources archéologiques eut initialement pour réponse des fouilles de sauvetage, entreprises sur une petite échelle et concernant surtout les édifices religieux et les centres urbains détruits par les bombardements. Ces fouilles-là ne différaient guère des fouilles programmées menées à l'époque par des institutions de recherche. Avec l'essor économique des années 1960 et 1970, cette stratégie aboutit à des fouilles de sauvetage sur une échelle sans précédent, accompagnant les projets de développement des infrastructures partout en Europe. Ce phénomène se poursuivit dans les années 1980, avec de puissantes organisations, agissant généralement sous le contrôle de l'État, engagées dans d'ambitieux chantiers archéologiques qui dominaient le travail sur le terrain. Ce travail, qui nécessitait des moyens importants, était la plupart du temps financé par les gouvernements, bien que, à l'occasion, quelques fonds puissent être alloués par les aménageurs.

Cependant, dans les années 1980, on commença à prendre conscience de la disparition rapide des ressources archéologiques sous les bulldozers: les fouilles de sauvetage ne pouvaient collecter qu'une infime partie de l'information, le reste étant irrémédiablement perdu. La profession reconnut alors la gravité de la menace pesant sur le patrimoine archéologique et prit conscience du besoin 
urgent de développer une approche différente qui permettrait de peser sur les décisions politiques et socioéconomiques, et de sensibiliser l'opinion publique afin d'en obtenir le soutien. Des conférences internationales, comme celles que le Conseil de l'Europe organisa à Florence, en 1984, puis à Nice, en 1987, suscitèrent une série de débats sur ces questions auparavant largement ignorées.

Deux idées principales ont émergé des débats: premièrement, pour assurer la protection optimale de ses ressources, l'archéologie doit faire partie intégrante du processus de planification. Deuxièmement, les idées engendrées par le mouvement écologiste devaient être adoptées et adaptées, le principe de "pollueur-payeur» se traduisant dans ce cas par le principe de «aménageur-payeur». Il parut normal, ou à tout le moins pas scandaleux, d'envisager que les aménageurs assument une partie du coût du travail archéologique induit par leur projet. En Europe, le débat international joua un rôle vital dans la mise en œuvre de ces changements.

Une initiative prise par le Conseil de l'Europe donna un puissant coup d'accélérateur à la diffusion de ces nouvelles idées concernant la gestion de l'environnement historique; ses membres introduisirent cette question au cœur de l'agenda politique et décidèrent de remplacer la convention de Londres de 1969, devenue obsolète, par une nouvelle convention intégrant ces idées nouvelles. La convention de Londres traitait des problèmes comme les fouilles illicites, la protection légale des sites, la diffusion de l'information, le contrôle et le classement des objets mis au jour. Sans remettre en question l'importance de ce qui précède, il était devenu clair qu'on avait ignoré les menaces les plus sérieuses pesant sur le patrimoine archéologique. Le Conseil des ministres en conclut qu'une révision de la convention existante s'imposait. ${ }^{3}$

\section{Le travail du comité. Les membres}

du Comité restreint d'experts sur l'archéologie et l'aménagement, auquel je participais, appartenaient tous à l'Europe occidentale et méridionale (Autriche, Belgique, Chypre, Danemark, France, Allemagne de l'Ouest, Grèce, Italie, Pays-Bas, Portugal, Espagne, Suède, Suisse, Turquie et Royaume-Uni).

1 Voir, par exemple,

Les Nouvelles de

l'archéologie, n82, 2000

(Willems, Theuws,

Monod, Randoin)

ou Public Archaeology,

$\mathrm{n}^{\circ}$ 2, 2002 (Demoule

et Thomas).

2 Une version anglaise

plus développée

de cet article sera publiée

ultérieurement.

3 Recommandation

$\mathrm{n}^{\circ} \mathrm{R}(89) 5$ concernant

la protection et la mise

en valeur du patrimoine

archéologique dans

le contexte de projets

d'aménagement urbains

et territoriaux.
4. Ce point est précisé
de façon explicite dans

le rapport explicatif

de la convention.
Si tous les membres du comité travaillaient dans la fonction publique, certains étaient diplomates ou avocats, mais ils étaient pour la plupart archéologues et spécialistes de la gestion du patrimoine.

D'emblée, la plupart d'entre nous décida d'agir en tant que groupe davantage intéressé par l'obtention des mesures les plus favorables à l'archéologie en général, et pas nécessairement à notre pays en particulier. Si un texte était proposé dont le contenu risquait d'être jugé inacceptable par les supérieurs politiques de certains participants, nous nous mettions d'accord sur une formule qui, nous l'espérions, survivrait à la censure de nos juristes reśpectifs tout en sauvegardant l'aspect qui nous paraissait le plus important.

La question se posa d'une définition du patrimoine archéologique. D'après l'article1.3, «sont inclus dans le patrimoine archéologique les structures, constructions, ensembles architecturaux, sites aménagés, témoins mobiliers, monuments d'autre nature, ainsi que leur contexte, qu'ils soient situés dans le sol ou sous les eaux». Ce n'était pas la proposition originelle pour ce paragraphe, mais même cette formule était trop inclusive aux yeux de certains pays du nord de l'Europe. En effet, elle pouvait être interprétée comme signifiant «le patrimoine bâti», lequel, à l'époque, était largement séparé du «patrimoine archéologique» sous au moins trois aspects. Premièrement, sous l'angle universitaire, parce que les bâtiments historiques sont étudiés dans des départements universitaires indépendants (histoire de l'art et/ou de l'architecture plutôt qu'archéologie). Deuxièmement, en termes juridiques, parce qu'il existe généralement des différences légales entre l'immobilier et le terrain - impliquant souvent des aspects financiers spécifiques: financements gouvernementaux indirects (réduction d'impôts) ou directs (subventions). Troisièmement, d'un point de vue organisationnel, parce que les deux types de patrimoine sont traités le plus souvent par des agences gouvernementales différentes. Il est intéressant de noter que cette dernière particularité a aujourd'hui largement disparu du fait des réorganisations dans de nombreux pays européens. L'approche universitaire est également devenue plus globale.

Cependant, la portée du nouveau traité restait une source de préoccupations. Certains pays du Nord estimaient que les dispositions du traité ne devaient pas s'appliquer au patrimoine bâti car cela impliquerait des recherches extrêmement coûteuses à chaque modification envisagée d'un monument historique. Les pays méridionaux avaient de leur côté de bonnes raisons de refuser de limiter la définition du patrimoine archéologique. Une porte de secours fut trouvée en déclarant, dans le paragraphe 1.2, que pour qu'une chose soit considérée comme un élément du patrimoine archéologique les principaux moyens d'information ayant servi à l'attester devaient être de nature archéologique. ${ }^{4}$ 
La signification exacte restait volontairement vague pour laisser chaque pays traiter du problème comme il l'entendait.

Les principaux enjeux. Les principaux enjeux étaient ceux qui avaient abouti aux articles 5, 6 et 9 de la convention, stipulant que l'archéologie doit participer aux politiques de planification, qu'elle doit être financée à travers les budgets des projets de développement, et que ses résultats doivent être communiqués au public.

Nous avons commencé par l'examen d'un avant-projet dû à Marc Gauthier, inspecteur général de l'Archéologie, en France. Une autre contribution, en provenance de Grande-Bretagne, nous fut également fort utile: Geoffrey Wainwright travaillait depuis quelque temps à l'amélioration de la gestion du patrimoine anglais en amenant les aménageurs à contribuer financièrement au travail archéologique induit par leur projet, sous peine de ne pas recevoir de permis de construire. En 1990, ce système fut adopté dans le cadre du Planning Policy Guidance note16, intitulé "Archaeology and Planning». Certaines de ces idées ont été reprises pour la convention européenne.

Un aspect intéressant fut l'utilisation de deux langues de travail, l'anglais et le français, lors des débats, ce qui a conduit le comité à davantage de précision dans le choix des termes et des phrases employés.

Parfois, aucune solution satisfaisante ne se présentait. Le meilleur exemple est une notion italienne et française des plus commodes, celle des «biens culturels», qui n'a pas d'équivalent anglais. Les interprètes avaient recours aux termes de cultural properties («propriétés culturelles»), un concept aux implications sensiblement différentes (Carman 2005). Malgré nos efforts, il subsiste des différences marquantes entre la version anglaise et la version française du texte définitif de la convention. On en trouve deux à l'article 6: le texte français est totalement adéquat quand il utilise les termes d' «archéologie préventive» (preventive archaeology), car c'est précisément l'objectif visé par l'ensemble du traité; le texte anglais utilise à mauvais escient les termes de rescue archaeology, en français «archéologie de sauvetage», qui représentent précisément ce que la convention visait à éviter en intégrant l'archéologie dans le processus de planification! En outre, dans la manière dont est formulé le paragraphe $2 \mathrm{~B}$, il est évident que le texte français est considérablement plus exigeant dans sa demande de «document scientifique de synthèse», alors que le texte anglais se contente d'un «résumé scientifique» (scientific summary record).

La traduction de la convention dans les diverses langues nationales fut accomplie, au-delà du français et de l'anglais, lors du second semestre 1991, alors que le comité avait achevé sa tâche. Nous étions rentrés chez nous et nous efforcions de convaincre nos ministères respectifs de participer à la conférence ministérielle de Malte de l'année suivante. En janvier 1992, pas moins de vingt pays signèrent la convention, dont plusieurs pays d'Europe de l'Est qui avaient décidé de se joindre au processus. Aujourd'hui, le nombre de signatures s'élève à trente-sept et le nombre de ratifications à vingt-six, ce qui en fait l'une des conventions les plus suivies du Conseil de l'Europe ( $c f$.www.coe.int, puis coopération culturelle, patrimoine).

Les suites. Le comité qui avait formulé le traité ne fut pas dissous. Il fut même maintenu pour travailler sur le «Plan européen pour l'archéologie» jusqu'en 1996, lorsque le Conseil de l'Europe se mit à manquer de fonds et à changer de priorités (ce qui revient au même). Il fallait en effet communiquer l'importance et l'utilité de l'archéologie à une audience européenne récemment élargie. Plusieurs pays se joignirent au comité: la Bulgarie, le Vatican, la Hongrie, l'Irlande, le Luxembourg, Malte, la Pologne et, plus tard, la Slovaquie.

Ce plan européen était engagé dans des activités et des projets pilotes correspondant aux standards fixés par la convention, ces projets étant menés par des groupes de travail incluant des spécialistes en provenance de différents pays. L'un de ces projets consistait à développer des «standards et des normes de données» pour les sites archéologiques, en collaboration avec le Cidoc $^{\mathbf{5}}$ (Conseil de l'Europe 1999a). Un deuxième projet pilote consistait à faire l'étude comparative de la situation de l'archéologie urbaine dans les États membres. Il en résulta une présentation générale d'une grande utilité comprenant des rapports sur vingt-deux pays (Conseil de l'Europe 1999b). L'ouvrage n'est pas très connu en raison d'une communication insuffisante du Conseil de l'Europe, mais il aurait mérité une meilleure diffusion. Une troisième activité, qui aboutit à une publication, consistait à établir un glossaire des termes de l'archéologie. Il était prévu de poursuivre ce projet avec le soutien financier de l'Union européenne et des États intéressés, mais il fut décidé de limiter sa réalisation à l'âge du Bronze. Un glossaire fut établi (Barber, Van Regteren Altena 1999) et traduit en anglais, en français, en danois, en hollandais et, plus tard, en roumain. D'autres langues devaient suivre et il était prévu d'ajouter la terminologie des autres périodes. Par manque de fonds (ou d'intérêt), le projet n'aboutit pas.

Le choix de lâge du Bronze pour le projet pilote émanait de la quatrième décision prise par le conseil des ministres de Malte. Il s'agissait de la «campagne de l'âge du Bronze» lancée par le Conseil de l'Europe pour faire connaître au public européen le rôle de l'archéologie. La Suède avait été à l'origine de cette proposition, faite lors du colloque de Cracovie de 1991, puis adoptée par le Conseil de l'Europe. Margaretha Björnstad, responsable des Antiquités suédoises, avait alors justifié ce choix parce que ni les Vikings ni les Romains ne pouvaient convenir pour promouvoir la notion d'un patrimoine commun 
européen. En revanche, les caractéristiques de l'âge du Bronze étaient suffisamment vagues et paneuropéennes, outre le fait qu'avait été exhumé un nombre important d'artefacts suffisamment intéressants pour attirer un large public. L'idée fut très bien accueillie par les eurocrates réunis à Strasbourg. En fait, toute cette campagne autour de ce que l'on appelait «l'âge d'or de l'Europe» illustre bien le rôle que pourrait jouer l'archéologie dans le processus politique d'unification de l'Europe, en contribuant à la création d'une identité et d'une conscience européennes. De fait, la gestion de cette campagne laissa à désirer, et son faible impact sur le public fut source de déception à Strasbourg. Il fut donc décidé de l'abandonner à l'issue d'une dernière conférence à Vérone, en Italie, vers la fin de 1996. Même si quelques activités furent maintenues jusqu'en 1999, la réunion de Vérone marqua la fin de l'intervention directe du Conseil de l'Europe dans la gestion du patrimoine archéologique.

De plus, sur le long terme, il est clair que les intérêts de l'archéologie doivent être représentés à Bruxelles, auprès de la Commission européenne, plutôt qu'à Strasbourg, au Conseil de l'Europe. Pour l'instant, le traité de la Communauté européenne n'inclut pas la culture; l'article 128 exclut précisément la culture des domaines de compétence de l'Union européenne, même si les lois et les subventions européennes exercent une influence croissante sur la recherche et sur la gestion des ressources archéologiques. Il n'en demeure pas moins possible pour l'Union européenne d'intégrer la convention de Malte dans la mesure où l'article 15 prévoit cette option. Ainsi, même si l'on peut prévoir des difficultés au niveau du traité, la convention pourra bien faire partie de l'arsenal législatif européen, ce qui renforcerait considérablement sa position. Il est plus probable que la gestion des ressources archéologiques au niveau européen dépende de plus en plus des lois européennes régissant d'autres domaines comme la planification de l'espace et la gestion de l'environnement. Une question reste ouverte: qui donc représente aujourd'hui les intérêts de l'archéologie, comme discipline et comme profession?

5 Le Comité

international pour la documentation de l'ICOM

(Conseil international des musées).
Barber M., Van Regteren Altena J. F. (dir.) 1999: Monuments de l'âge du Bronze. Glossaire multilingue des termes archéologiques, Strasbourg.

Carman J. 2005: Against Cultural Property. Archaeology, Heritage and Ownership, Londres.

Conseil de l'Europe

1969: Convention européenne pour la protection du patrimoine archéologique, Strasbourg (Série des traités du Conseil de l'Europe 66).

1987: Archaeology and Planning. Report of the Florence Colloquy, Strasbourg (Architectural heritage reports and studies 5).

1989: Archaeology and major public works. Report of the Nice Colloquy, Strasbourg (Architectural heritage reports and studies 12).

1992: Convention européenne pour la protection du patrimoine archéologique (révisée), Strasbourg (Série des traités du Conseil de l'Europe 143).

1999a: Fiche d'indexation minimale pour les sites archéologiques, Strasbourg.

1999b: Rapport sur la situation de l'archéologie urbaine en Europe. Strasbourg.

Willems W. J. H., VAN DEN Dries M. (dir.) 2007: Quality Management in Archaeology, Oxford. 